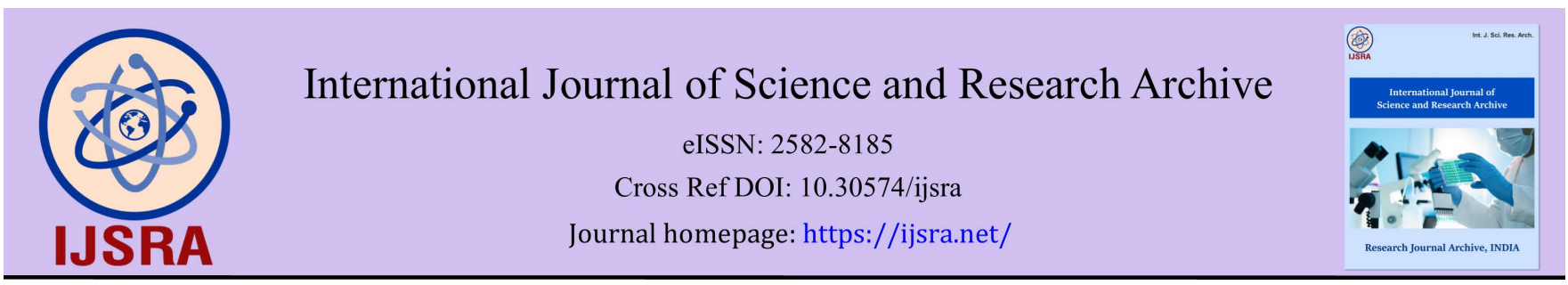

(REVIEW ARTICLE)

Check for updates

\title{
Green energy and its challenges in the Brazilian Amazon: An analysis in the state of Pará, Brazil
}

\author{
Fabricio Quadros Borges ${ }^{1, *}$ and Desiree Moraes Zouain ${ }^{2}$ \\ ${ }^{1}$ Department of Public Management, Federal Institute of Pará, Brazil. \\ 2 Department of Research and Development, Institute of Energy and Nuclear Research, São Paulo, Brazil.
}

International Journal of Science and Research Archive, 2021, 03(02), 224-229

Publication history: Received on 21 September 2021; revised on 24 October 2021; accepted on 26 October 2021

Article DOI: https://doi.org/10.30574/ijsra.2021.3.2.0167

\begin{abstract}
The aim of this investigation is to analyze the main green energies for electricity generation in the Brazilian Amazon, especially given the location specificities of the State of Pará, Brazil. Electric energy is an invaluable resource for the development of populations and green energy comprises viable alternatives to sustain this development. The methodology of this study is classified in terms of the approach to the problem as a qualitative research and in terms of its nature, as a theoretical study. The green energies analyzed in the State of Pará, Brazil, were: hydro, biomass, solar and wind. The study concluded that the electricity generation base in the State of Pará should continue to be the hydro base; however, Pará should increase the proportion of use of sources from biomass and the sun.
\end{abstract}

Keywords: Electricity Generation; Environment; Development; Sustainability

\section{Introduction}

The availability of electricity sources has been the subject of wide discussion by governments in several countries that face permanent challenges as they seek to identify electricity generation sources capable of meeting their demands in a sustainable manner. In this perspective, green energies are presented. Green energy or clean energy comprises any energy generated from natural and renewable raw materials, such as air, water, sun and heat from the ground.

The generation of electric energy in Brazil is mainly based on the use of water power through hydroelectric plants. This picture is based on the abundance of natural resources at low costs in relative terms, notably in the Amazon region. The question that arises for the future is whether the country will be able to maintain this comparative advantage and still guarantee the population wide access to this input. Electricity generation in the country grew at an average annual rate of $5 \%$ throughout the 1990 s and 2000s [1]. During this period, the water source accounted for more than $80 \%$ of the total electricity generated in the country. Not only does hydroelectricity dominate electricity generation in Brazil, but also large plants dominate the sector, as there are around 450 hydroelectric plants in operation [2].

In the State of Pará, located in the Brazilian Amazon region, which has great natural potential and a notable potential for exporting electricity, the challenges are not only associated with ensuring the availability of this input. Reducing inequalities and poverty, universalizing access to electricity and minimizing the costs and environmental damage arising from the use of water sources are challenges that are imposed in light of the reality of Pará. Its important hydroelectric potential from large projects implies sudden impacts on the hydrological cycle and changes in the environment in general [3].

\footnotetext{
${ }^{*}$ Corresponding author: Fabricio Quadros Borges

Department of Public Management, Federal Institute of Pará, Brazil.

Copyright (@ 2021 Author(s) retain the copyright of this article. This article is published under the terms of the Creative Commons Attribution Liscense 4.0.
} 
In view of this scenario, it is highlighted that the State of Pará needs to be prepared to develop other green energies for generation, complementary to hydropower, in view of facing the insecurity in the supply of electricity and the challenge of environmental issues. Green energy generation projects from biomass, sun and wind should be the object of studies in order to know the limits and potential of these generation systems. Projects to implement new alternative sources of electricity generation have the possibility of promoting employment and income, in order to increase the possibilities of accessing information and improving living conditions.

In this sense, this investigation intends to question the potentials and limitations of green energies in view of the locational specificities of the State of Pará. Given this context of approach, it is observed that the technological and economic limits for the high penetration of green energies in the system of Electric power still exists, for the most part, relating to the storage of grid flexibility and power needed to incorporate electricity generation from intermittent sources into the transmission grid [4]. And the continental proportions of the State of Pará contribute even more to this difficult challenge. This investigation, in addition to this introduction, is composed of four parts, namely: methodology, theoretical framework, result and discussions, and conclusion.

\section{Methodology}

The investigation is classified in terms of the approach to the problem as a qualitative research and in terms of nature, as a theoretical study. It is qualitative, as it aims to provide a better vision and understanding of green energy. It is theoretical in nature in that it analyzes the applicability of these green energies in the reality of the Brazilian Amazon, especially in the State of Pará. This section is divided into two parts, namely Place of study and Research techniques.

\subsection{Place of study}

The spatial cutout used for the development of the research is the State of Pará, which comprises a geographical area of $1,245,870,798 \mathrm{~km}^{2}$, representing $14.7 \%$ of the Brazilian territory. Pará is located in the Brazilian Amazon. The estimated population of Pará is 8,690,745 inhabitants, which gives it a density of 6.07 inhabitants $/ \mathrm{km}^{2}$.

The public electricity distribution service in the State is a concession of the Equatorial Energia Group. The generation market is the domain of Central Elétricas do Norte (Eletronorte). In Pará, 98.8\% of permanent Pará households are supplied with electricity, leaving the challenge of total supply conditioned to the expansion of distribution services to rural households, where the coverage of electricity supply in households reduces to approximately $95 \%$.

\subsection{Research techniques}

The methodology was divided into three stages: data collection, treatment and analysis. Data collection was carried out through a bibliographic survey, through books and periodicals that address the researched conceptual categories. The collection process took two directions: the applicability of green energies and the specificities of the State of Pará, as recipient of these energies. Data processing sought to associate economic, social and technological aspects and considered each of the main green energy. They are: hydro, biomass, solar and wind. Data analysis, in turn, aimed to examine the intricacies of the application of green energy in the face of the locational specificities of the State of Pará. The analysis aimed to elucidate the potential and limitations of each researched green energy.

\section{Theoretical framework}

This section will cover green energy. Among the main ones, hydro, biomass, solar and wind energy can be highlighted. These energies can generate electricity from different technologies.

\subsection{Hydropower}

The generation of electricity from water sources is characterized as renewable energy. Hydroelectric plants are based on simple fundamentals. Turbines extract energy from the flowing water of rivers, using it to drive electrical generators (a system that transforms mechanical energy into electricity through the kinetic force, which is due to the speed of water flow, and the potential force, which refers to the fall water) [5]. The dam exerts control over the amount of water flowing through the turbines, so the plant's productivity is regulated according to demand.

The State of Pará, holder of numerous waterfalls and extensive rivers, provides a vast storage capacity in the reservoirs. The growing trend in consumption is also of no concern in the long term as, according to the National Energy Balance BEN (2007), the country has a reserve of $144 \mathrm{GW}$ of firm energy/year from hydroelectric sources and uses $34 \mathrm{GW}$, that is, only $23.6 \%$ [6]. 
However, this panorama that has been taking shape for decades in Pará, definitely does not contribute, in the medium or long term, to the reduction of greenhouse gas emissions by its electrical matrix. The water source through large projects, characterized as clean, causes serious and extensive impacts on the hydrological cycle and changes in the environment in general. The results show the disappearance of species of fauna and flora, loss of quality of life for the affected populations and threats to the existence of various social groups.

The emission of greenhouse gases represents another serious problem caused by large hydroelectric projects in Pará. In studies measuring $\mathrm{CO}^{2}$ carbon dioxide emissions from water sources in the state, it was found that between 1995 and 2005, this source of energy it released about 90 million tons of greenhouse gases into the atmosphere [7]. In comparative terms with deforestation in Pará, it appears that emissions from deforestation between 1999 and 2008, which used a methodology for calculating pollutant gas emissions by state, point to about 6 million tons of $\mathrm{CO}^{2}$ from of the Pará territory [8]. The much lower amount of emissions from deforestation when compared to emissions from reservoirs draws attention to the intensity of the environmental impacts of the water source in the State.

The closing of a river by a dam causes a structural change where the water passes from a current system, called lotic, to a still water system, called lentic. With the reservoir in place, several tons of organic matter decompose at the bottom of the dam, releasing carbon dioxide and methane [9]. Lentic waters favor the appearance of aquatic plants (macrophytes). Thus, there is the emission of carbon dioxide from the decomposition of organic matter above water. Methane, in turn, is produced when decomposition occurs at the bottom of the reservoir, with soft green matter, such as macrophytes [9].

The large areas of mud exposed in the dry period allow for the growth of soft vegetation which, when flooded in the flood, under anoxic conditions becomes methane factories [9]. Methane would be released when water pressure suddenly drops as water emerges from the turbines. Therefore, the emission of carbon from the decomposition of organic matter and macrophytes in dams ends the idea that hydroelectric plants produce clean energy. Considering that the macrophytes reproduction condition is excellent in the dams, there is nothing that indicates a reversal of the greenhouse gas emission process by the Pará dams in the long term.

Another problem related to macrophytes stands out, which is the possibility of increasing the population density of Culicidae. Specifically in Tucuruí, in the State of Pará, the expansion of the genera Anopheles and Mansonia was reported, in addition to other insects, which use macrophytes, especially Salvinia, Eicchornia and Pistia, to reproduce [10]. According to the author, these plants occupy approximately $30 \%$ of the dam's surface. The Curuá-una dam, also in the state of Pará, is covered with macrophytes, which cover $27 \%$ of the area, promoting the development of mosquitoes and snails that transmit schistosomiasis, a disease that was unknown in the region of the municipality of Santarém in Pará. In this sense, it is not surprising to find that concerns about the economic viability of large hydroelectric plants and the significant environmental impacts arising from the construction of dams and reservoirs have reduced the world growth rate of this type of generation to a modest rate of $1.5 \%$ per year [5].

\subsection{Biomass energy}

The State of Pará has a significant potential for biomass from firewood and residues. The State is the third largest beneficiary of forest species in Brazil, consequently generating a large amount of residues, which represents a significant potential for using this biomass for energy purposes. Firewood, understood as a set of tree trunks, pieces and splinters of wood and sticks, has been highlighted in the 2000s as an important source of primary energy in Pará. In 2005 , firewood accounted for $47.6 \%$ of the supply domestic primary energy [6], however, this input has still incipient use in the generation of electricity in the State.

Biomass is a type of matter that feeds steam power plants for electrical generation from a process of burning elements accumulated in a given ecosystem. Among the most used materials are sugarcane bagasse and woody materials. The burning of biomass causes the release of carbon dioxide into the atmosphere, however, this compound was previously absorbed by the plants that originated the fuel, which provides a zero $\mathrm{CO}^{2}$ emission balance [11].

Most of the biomass potential, from woody material, is found in the Pará municipalities of the National Interconnected System, which can be used for self-production, being also viable in many cases the transport of biomass to municipalities that are part of the isolated system. In addition to firewood, forestry residues, which comprise all the organic forestry material that is left over after the firewood is removed, and woody residues can also be used as primary energy, especially residues from sawmills. Pará has about 4,000 sawmills formally registered and part of the waste from these sawmills has no destination, being burned in the open or thrown into rivers, causing significant environmental damage [12]. 
As for the costs of using biomass, it is observed that, taking into account that steam plants have lower operating costs compared to diesel plants, and due to their technical and economic attractiveness, thermoelectric plants can be implemented successfully in Pará [12]. Many mesoregions present sustainability for the supply of biomass. The Marajó mesoregion was the one that presented the best possibility of using biomass within its own area, as it required little biomass from another mesoregion, as it has a potential for generating energy with biomass around 16 MW. It is also important to add that materials such as paper already used, cardboard packaging, leftover logs and trees lost by plant exploitation, branches from tree pruning in urban areas and sawdust, quite common in the wood industry in Pará, can also be widely used as food for thermoelectric power plants. It is also important to mention that these materials must be near thermoelectric plants or on strategic routes with easy access. Biomass comprises a low-cost and less polluting source, which can represent a very reasonable alternative if established on a medium scale in the State of Pará.

\subsection{Solar energy}

The solar source comprises another alternative for generating electricity in Pará. The use of the sun in the generation of electricity can occur in two ways: indirectly, generated by the use of heat that feeds a thermoelectric plant, and directly, generated by the use of panels photovoltaics. Photovoltaic generation has had much more application, especially for powering small isolated systems, for pilot projects and for the electrification of solitary equipment [13]. This type of energy source is considered clean, renewable and inexhaustible. The main disadvantages of the solar source are: the high cost of implementing photovoltaic panels, which are very expensive to enable large-scale electricity production, and their irregularity in the form of uniform distribution, which requires large collection areas and storage systems. However, photovoltaic conversion appears as an alternative supply, in order to promote: the generation of local jobs; the maintenance of revenue from the production and sale of energy in the region itself; and a self-sustained development process [14]. It is noteworthy, however, that the simple photovoltaic conversion does not allow the development of productive activities. It is necessary to apply other strategic mechanisms capable of spreading a process of socioeconomic development consistent with the demands and local economic characteristics.

The potential for generating jobs from solar energy can be demonstrated through the composition of the segments of the production chain, from the processing of quartz (non-metallic mineral from which silicon is extracted, input for photovoltaic panels) to production and distribution solar energy itself. With regard to the processing of quartz, it is noteworthy that, according to the National Department of Mineral Production [15], Pará has quartz reserves in the order of 1,627,994 t, notably in Breu Branco and Marabá, municipalities belonging to the mesoregion Southeast of the State, which is equivalent to $36.6 \%$ of the national reserves of this mineral. In the state of Pará, the use of the solar source is indicated thanks to the large amount of solar radiation available throughout the year. The Amazon receives, on average, 400 calories per square centimeter daily, of which around 120 refer to the territory of Pará, an amount that is many times greater than the demand of the State of Pará [16]. With regard to the potential for generating jobs, it is worth noting that biomass also has potential conditions for generating these jobs. The capacity of jobs directly and indirectly generated by an average electricity generation plant from biomass is 300 jobs [11].

\subsection{Wind energy}

Wind energy comprises another possibility of an alternative source of generation in Pará. It comes from a technology that uses the force of the wind, which, in turn, operates turbines connected to electricity networks. This type of energy source, because it is classified as a renewable source, tends to grow notably in developed countries, as it is of a renewable nature, has a low cost of externalities, does not burn fossil fuels and does not emit polluting gases that cause the greenhouse effect. Among the disadvantages of this source, the following stand out: the alteration of the landscape when its infrastructure was implemented, consisting of propellers and towers, the emission of low-frequency noise, the threat to migratory routes of birds due to the use of large rowed propellers and the unproductiveness of this source in some regions due to the inconstancy of winds, their low intensity and wasted energy in the occurrence of heavy rains.

In the territory of Pará, winds are registered at speeds between 3.5 and 4 meters per second, at a height of 50 meters. If a $300 \mathrm{~kW}$ wind turbine, with a rotor diameter of 33.4 meters, were installed from that speed, it would produce a maximum power between 8.9 and $15 \mathrm{~kW}$ [17]. For the author, these speeds would be enough to operate wind turbines with speeds from $3 \mathrm{~m} / \mathrm{s}$. However, the wind speed between 3.5 and $4 \mathrm{~m} / \mathrm{s}$, dominant in the territory of Pará, is among the sufficient to only operate the wind turbines in minimal conditions and it is admitted that these turbines would never reach an optimal performance, which it does not justify a broad program for the implantation of turbines in the State [17]. The State of Pará has a large part of its territory with low wind density, which does not recommend the use of this type of energy on a large or even medium scale [18]. Pará still presents a disadvantage for the use of wind energy: the unproductiveness of this source in some micro-regions of the State due to the inconstancy of winds, their low intensity and energy waste in the event of heavy rains. Rains are abundant in Pará, with annual precipitation ranging from 1,500 $\mathrm{mm}$, in the south of the state, to 3,500 mm - 4,000 mm, in the estuary of the Amazon River [19]. 


\section{Results and discussion}

The process of diversifying energy sources in the State of Pará, through green energies, has two roles. The first is characterized by a concrete effort to solve environmental problems arising from electricity generation sources in the State, insofar as it reduces atmospheric emissions of polluting gases. The second is related to the fight against poverty through the generation of jobs and the offer of opportunities from a local production chain of energy technology that is not dependent on imports.

It is also important to point out, opportunely, that the forms of use and distribution of electricity generated in Pará also interfere in the State's development process. It is registered that, approximately, half of the electric energy consumption in the State of Pará is directed to electro-intensive ones [20].

The subsidy provided by the energy supply contracts produced by the Tucuruí Plant represents for Eletronorte a loss of approximately R 370 million per year [14]. Developing an infrastructure in Pará associated with the profile of these industries is equivalent to adopting a policy of not adding value to goods produced in the state and still bearing the social and environmental costs of this generation, which hinders the evolution of the process of socioeconomic development in the state of Pará. This panorama, in addition to making its population the holder of the broad social and environmental impacts arising from the construction of these large projects, places the State in a condition of submission to the logic of capital [11]. This occurs as it excludes several communities from Pará from social benefits in favor of expanding the national GDP in the short term.

Regarding the water source, it was observed that the State of Pará records a high performance in the economic, social and technological dimensions, and a medium performance in the environmental dimension. Which indicates its use in the State.

In relation to biomass, the study found that Pará presents high performance for the economic dimension, medium performance for the social dimension, medium performance for the environmental dimension and medium performance for the technological dimension. Its use is indicated. The State is the third largest beneficiary of forest species in Brazil, consequently generating a large amount of waste, which represents a significant potential for using this biomass for energy purposes.

With regard to the solar source, it was observed that the State presented low performance in the economic dimension, medium performance in the social dimension, high performance in the environmental dimension and medium performance in the technological dimension. The use is indicated for Pará thanks to the enormous amount of solar radiation that the State has throughout the year.

In relation to the wind source, the work found that Pará presents low performance for the economic dimension, low performance for the social dimension, high performance for the environmental dimension and low performance for the technological dimension. Its use is not indicated. The state has a large part of its territory with low density of winds, less than $4 \mathrm{~m} / \mathrm{s}$, and their inconstancy.

Finally, it should be noted that the planning effort in the use of green energies that are more committed to the competitive and environmental challenges presented by the international panorama today, must be the result of a sustainable and integrated national plan. If the Federal Government's planning is not committed to the need to transform the national electricity matrix and the political discourse remains committed to the generation of GDP in the short term, the growth of electricity consumption in Brazil will inevitably indicate the need to build new ventures hydroelectric power plants in Pará, which would substantially compromise the strategic use of other green energies.

\section{Conclusion}

Faced with this challenge of green energies, this study questioned the potential and limitations of green energies in view of the locational specificities of the State of Pará. In this perspective, it verified the feasibility of inserting the main green energies for the generation of electricity with increasing diffusion in the verified realities, in the economic, technological and natural specificities of the State of Pará. The intention was to improve the strategic positioning of the composition structure for the use of electricity generation sources. Thus, the sources were evaluated: hydro, biomass, solar and wind.

The study concluded that the electricity generation base in the State of Pará should continue to be the hydro base; however, Pará should increase the proportion of use of sources from biomass and the sun. The limitations of this study 
lie in its theoretical nature, which lacks further empirical verification. Future investigations should be directed towards the development of plans and estimates for the implementation and use of green energies based on biomass and the sun, for the State of Pará.

\section{Compliance with ethical standards}

\section{Disclosure of conflict of interest}

The authors have no conflict of interests.

\section{References}

[1] Tolmasquim MT, Guerreiro AE, Gorini R. Prospective view of the Brazilian energy matrix: energizing the country's sustainable development. Brazilian Journal of Energy. 2007; 13(1).

[2] Ministry of Mines and Energy - MME. Belo Monte will produce energy with respect for the environment. Brasilia, 2012.

[3] Junk WJ, Mello JASN. in. Ecological impacts of hydroelectric dams in the Brazilian Amazon basin. Study Av., São Paulo. nineteen ninety; 4(8).

[4] Gaetani M, Huld T, Vignati E, Monforti-Ferrario F, Dosio A, Raes F. The near future availability of photovoltaic energy in Europe and Africa in climate-aerosol modeling experiments. Renewable and Sustainable Energy Reviews. 2014; 38: 706-716.

[5] Walisiewicz M. Alternative energy: solar, wind, hydroelectric and biofuels. São Paulo: Publifolha. 2018.

[6] Energy Balance of the State of Pará (2007). BEEP Belém: BEEPA. 2007.

[7] Santos MA. Inventory of greenhouse gas emissions from hydroelectric power plants. 2000. $523 \mathrm{f}$. Coordination of Graduate Engineering Programs. (Doctoral thesis). Rio de Janeiro: UFRJ. 2000.

[8] Aguiar AP, Ometto JP, Valeriano D, E Nobre CA. Calculation of $\mathrm{CO}^{2}$ emissions from deforestation - Methodology for the Amazon. In: VII Technical Scientific Seminar on Deforestation Data Analysis. Brasilia, May 20 and 21, 2010.

[9] Fearnside PM. The Amazon forest and global changes. Manaus: INPA. 2004.

[10] Marin REA. Amazon: The Ecological Cost of Hydroelectric Power Plants. In: Magalhães, S.B.; Britto, R. C. E Castro E. R. (Org.) Energy in the Amazon. Belém. Upeg/Ufpa/Unamaz. 2000.

[11] Borges FQ, Zouain DM. The electrical matrix and its position in sustainable development in the state of Pará. Revista Planning and Public Policies, Brasília: IPEA. 2010; 35: 187-221.

[12] Padilha JL, Rendeiro G, Brazil ACM, Santos RE, de E Pinheiro, G. Potential for electricity generation in the State of Pará: using biomass from the wood sector. Biomass and Energy Magazine. 2005; 2(4): 267-284.

[13] Reis LB, Fatigas EAA, Carvalho CE. Energy, natural resources and the practice of sustainable development. São Paulo: Manole. 2005.

[14] Bermann C. Energy in Brazil: for what? For whom? Crisis and alternatives for a sustainable country. São Paulo: Library of Physics. 2013.

[15] National Department of Mineral Production. Brazilian Mineral Yearbook. Brasilia: MME. 2005.

[16] Marques Filho A. de O. and Dallarosa, RG. Interception of solar radiation and leaf area distribution in terra firme forest in central Amazonia. Acta Amazônica Magazine. Manaus. 2004.

[17] Silva MVM. The exclusionary dynamics of the Pará electric system. São Paulo: EP/FEA/IEE/IF-USP. 2005.

[18] Rendeiro G. Experimental analysis of assai palm biomass for energy power generation. In: 7th International Conference on Energy for a Clean Environment. Lisbon. 2003.

[19] Brazilian Institute of Geography and Statistics - IBGE. Indicators. Rio de Janeiro. 2018.

[20] Borges Fabricio Q, Borges Fabrini Q, Vasconcelos MR. Public Energy management and decision-making model: a proposal based on energy sustainability indicators. Global Journal of Human-Social Science. 2021; 21(3). 